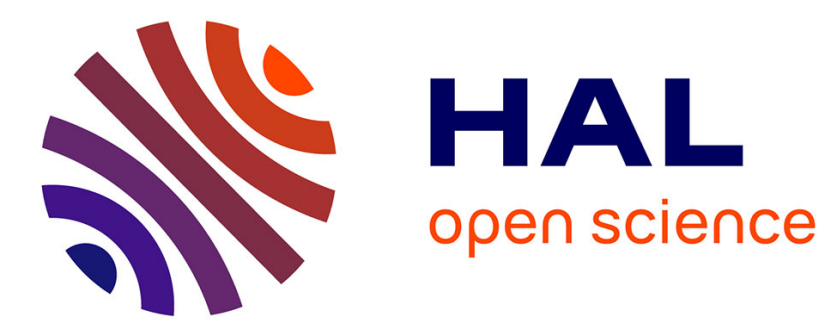

\title{
Sound attenuation optimization using metaporous materials tuned on exceptional points
}

\author{
Lei Xiong, Benoit Nennig, Yves Aurégan, Wenping Bi
}

\section{To cite this version:}

Lei Xiong, Benoit Nennig, Yves Aurégan, Wenping Bi. Sound attenuation optimization using metaporous materials tuned on exceptional points. Journal of the Acoustical Society of America, 2017, 142 (4), pp.2288 - 2297. 10.1121/1.5007851 . hal-01622702

\section{HAL Id: hal-01622702 https://hal.science/hal-01622702}

Submitted on 24 Oct 2017

HAL is a multi-disciplinary open access archive for the deposit and dissemination of scientific research documents, whether they are published or not. The documents may come from teaching and research institutions in France or abroad, or from public or private research centers.
L'archive ouverte pluridisciplinaire HAL, est destinée au dépôt et à la diffusion de documents scientifiques de niveau recherche, publiés ou non, émanant des établissements d'enseignement et de recherche français ou étrangers, des laboratoires publics ou privés. 


\title{
Sound attenuation optimization using metaporous materials tuned on exceptional points
}

\author{
Lei Xiong, ${ }^{1}$ Benoit Nennig, ${ }^{2, a)}$ Yves Aurégan, ${ }^{1}$ and Wenping $\mathrm{Bi}^{1}$ \\ ${ }^{1)}$ Laboratoire d'Acoustique de l'Université du Maine, Unité Mixte de Recherche CNRS 6613, Avenue Olivier Messiaen, \\ 72085 Le Mans Cedex 9, France \\ ${ }^{2)}$ Institut supérieur de mécanique de Paris (SUPMECA), Laboratoire Quartz EA 7393, 3 rue Fernand Hainaut, \\ 93407 Saint-Ouen, France.
}

(Dated: 24 October 2017)

\begin{abstract}
A metamaterial composed of a set of periodic rigid resonant inclusions embedded in a porous lining is investigated to enhance the sound attenuation in an acoustic duct at low frequencies. A transmission loss peak is observed on the measurements and corresponds to the crossing of the lower two Bloch modes of an infinite periodic material. Numerical parametric studies show that the optimum modal attenuation can be achieved at the exceptional point in the parameter plane of inclusion position and frequency, where the two lower modes merge.
\end{abstract}

The following article appeared in J. Acoust. Soc. Am. 142, 2288 (2017) and may be found at http: //asa.scitation.org/doi/10.1121/1.5007851.

PACS numbers: 43.20.Mv, 43.50.Gf, 43.28.Py

Keywords: duct acoustics;metamaterials;porous materials;Bloch waves;exceptional points

\section{INTRODUCTION}

Acoustic treatments are often used to limit noise propagation in acoustic waveguides such as ventilation systems, exhaust devices, and aircraft engines. In the last situation, the acoustic liners are generally made with a perforated sheet backed by a honeycomb ${ }^{1}$. This kind of material has good attenuation properties only in a narrow frequency band, but their main advantages are their mechanical robustness and their capability to resist to harsh conditions i.e., they constitute the reference solution in a turbofan engines.

For the future aircrafts, there is a need for efficient treatments at low frequencies with small thickness. That is why other materials, like micro-perforated plate ${ }^{2,3}$, extended Helmholtz resonator ${ }^{4}$ or slow sound materials ${ }^{5-7}$, which uses folded side branch quarter wavelength resonators to reduce the effective compressibility of the fluid, have been used to enhance sound attenuation in a duct with a grazing flow.

For some other applications, porous materials are often used $^{8-13}$ to reduce the noise emission, e.g., in the ventilation systems and the Auxiliary Power Units (APU) of aircrafts. Porous materials generally offer a wider absorption/attenuation band ${ }^{14}$ and the recent progresses of metallic foam open the door for porous materials solution in an aggressive environment. Nonetheless, they suffer from a lack of absorption efficiency at low frequencies, comparing to their efficiency at higher frequencies. The usual way, to solve that problem for absorbing panel,

\footnotetext{
a) Corresponding author.; Electronic mail: benoit.nennig@supmeca.fr
}

is by multi-layering ${ }^{15,16}$. Other alternative solution is to embed periodic inclusions ${ }^{17,18}$ or subwavelength resonators $^{19-23}$ to create metaporous materials (resonant inclusions embedded in a porous matrix). Thanks to this approach, it is possible to get a total absorption ${ }^{24}$ below the quarter wavelength frequency and to widen the absorption in a specified frequency range. The principle is that the energy is trapped and dissipated between the rigid inclusions and the rigid backing or in the inclusions themselves.

To the best of authors' knowledge, such metaporous materials have not been tested for duct applications. The multimode incidence of sound waves which depends on the duct dimensions makes the design of appropriate acoustic treatments more complex and less understood than for panels dedicated to room acoustic corrections.

The aim of this paper, is i) to illustrate the potentialities of periodic rigid inclusions embedded in a porous material for waveguide attenuation and ii) to link the optimal modal attenuation with higher modes interaction such as double roots of the dispersion equation ${ }^{25-27}$ or exceptional points ${ }^{28-35}$. The interest of such configurations has been shown in a preliminary numerical work 36 for basic $2 \mathrm{D}$ inclusions. We focus here on a 3D setup from both experimental and numerical point of view.

The present paper is organized as follows. After presenting Bloch wave and the finite element (FE) formulation in Sec. II, a description of the experimental protocol is presented in Sec. III, and finally results are compared. 


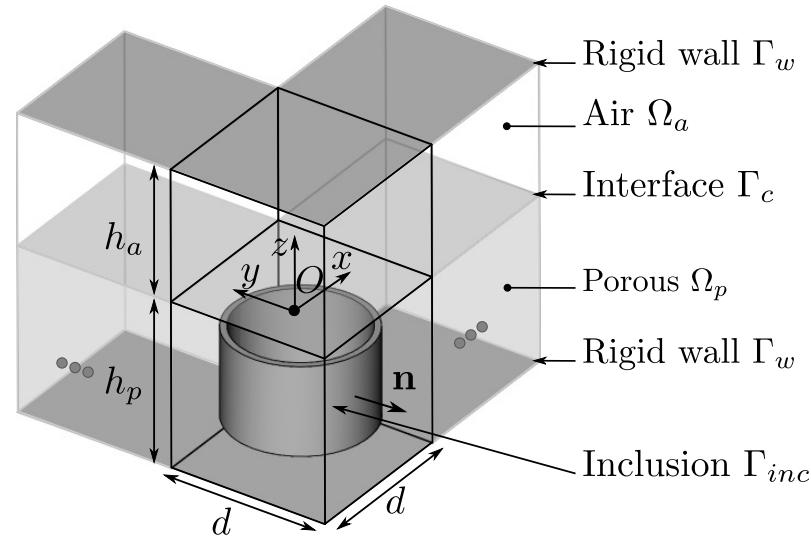

FIG. 1. Geometry of the problem.

\section{BLOCH WAVE IN A DUCT}

\section{A. Problem statement}

We consider time harmonic acoustic wave propagation $\left(\mathrm{e}^{-\mathrm{i} \omega t}\right)$ in a $3 \mathrm{D}$ infinite periodic waveguide $\Omega_{a}$ of height $h_{a}$ lined with a porous material $\Omega_{p}$ of height $h_{p}$ with embedded rigid inclusions, as described Fig. 1. It is noteworthy that air cavity is present inside the inclusion (see sec. III C). The unit cell is a rectangular parallelepiped of height $H=h_{a}+h_{p}$ and width $d$. The elementary cells are aligned on a regular $\mathbf{d}$-periodic grid with $\mathbf{d}=[d, d, 0]^{\mathrm{t}}$.

The skeleton of the porous material is supposed to be infinitely rigid, thus the Johnson-Champoux-Allard (JCA) equivalent fluid model ${ }^{15}$ is used to get the equivalent bulk modulus $K_{p}(\omega)$ and density $\rho_{p}(\omega)$ (see Appendix A for details). The sound speed is then given by the ratio $c_{p}(\omega)=\sqrt{K_{p}(\omega) / \rho_{p}(\omega)}$. In the air, the density is taken as $\rho_{a}=1.2 \mathrm{kgm}^{-3}$ and sound speed is $c_{a}=340$ $\mathrm{ms}^{-1}$

In this study only no mean flow case is considered. In each domain $\Omega_{\alpha}(\alpha=a, p)$, the pressure field is governed by the Helmholtz equation

$$
\Delta p_{\alpha}+k_{\alpha}^{2} p_{\alpha}=0
$$

with the wavenumber $k_{\alpha}=\omega / c_{\alpha}$, defined as the ratio between the angular frequency $\omega$ and the acoustic wave sound speed $c_{\alpha}$.

Air and porous media are coupled together at the interface $\Gamma_{c}$ between the two waveguides and the interface at the inclusion opening. Here we ensure the continuities of the pressure $p_{\alpha}$ and the normal velocity $\mathbf{v}_{\alpha} \cdot \mathbf{n}$ where

$$
\mathbf{v}_{\alpha}=\frac{1}{\mathrm{i} \omega \rho_{\alpha}} \nabla p_{\alpha}
$$

On the rigid walls $\Gamma_{w}$ and $\Gamma_{\text {inc }}$, the normal velocity vanishes $\mathbf{v}_{\alpha} \cdot \mathbf{n}=0(\alpha=a, p)$.

\section{B. Bloch modes computation}

As the governing equations, the boundary conditions, and the geometry are $\mathbf{d}$-periodic, it follows from the Bloch theorem that the solutions are Bloch waves,

$$
p_{\alpha}(\mathbf{x})=\hat{p}_{\alpha}(\mathbf{x}) \mathrm{e}^{\mathrm{i} \mathbf{k}_{B} \cdot \mathbf{x}},
$$

i.e. the pressure field can be split into a $\mathbf{d}$-periodic field $\hat{p}_{\alpha}(\mathbf{x})$ modulated by a plane wave involving the Bloch wavevector $\mathbf{k}_{B}$. The real part of $\mathbf{k}_{B}$ measures the change in phase across the cell and its imaginary part the attenuation. We put $\mathbf{k}_{B}=k_{B} \boldsymbol{\kappa}$ where the unit vector $\boldsymbol{\kappa}=\mathbf{k}_{B} / k_{B}$ stands for the propagation direction and $k_{B}$ is the wavenumber. In the following, $\boldsymbol{\kappa}$ is supposed to be aligned on the waveguide axis and is fixed to $[1,0,0]^{\mathrm{t}}$. This decomposition holds if the real and imaginary parts of $\mathbf{k}_{B}$ are collinear.

Combining with the homogeneous problem from Eq. (1), this yields an eigenvalue problem for the Bloch wave and the Bloch wavenumber $k_{B}$,

$$
\Delta \hat{p}_{\alpha}+2 \mathrm{i} k_{B} \kappa \cdot \nabla \hat{p}_{\alpha}+\left(k_{\alpha}^{2}-k_{B}^{2}\right) \hat{p}_{\alpha}=0 .
$$

The associated weak formulation is obtained after multiplying Eq. (4) by a periodic test function $\hat{q}_{\alpha}(\alpha=a, p)$ and integrating by parts over a unit cell, namely

$$
\begin{aligned}
& \frac{1}{\rho_{\alpha}} \times\left\{-\int_{\Omega_{\alpha}} \nabla \hat{q}_{\alpha} \cdot \nabla \hat{p}_{\alpha} \mathrm{d} \Omega+k_{\alpha}^{2} \int_{\Omega_{\alpha}} \hat{q}_{\alpha} \hat{p}_{\alpha} \mathrm{d} \Omega\right. \\
& \quad+\mathrm{i} k_{B} \int_{\Omega_{\alpha}}\left(-\nabla \hat{q}_{\alpha} \cdot\left(\boldsymbol{\kappa} \hat{p}_{\alpha}\right)+\left(\boldsymbol{\kappa} \hat{q}_{\alpha}\right) \cdot \nabla \hat{p}_{\alpha}\right) \mathrm{d} \Omega \\
& \quad-k_{B}^{2} \int_{\Omega_{\alpha}} \hat{p}_{\alpha} \hat{q}_{\alpha} \mathrm{d} \Omega \\
& \left.\quad+\int_{\partial \Omega_{\alpha}} \hat{q}_{\alpha}\left(\frac{\partial \hat{p}_{\alpha}}{\partial n}+\mathrm{i} k_{B}(\boldsymbol{\kappa} \cdot \mathbf{n}) \hat{p}_{\alpha}\right) \mathrm{d} \Gamma\right\}=0 .
\end{aligned}
$$

The global formulation is obtained by summing the weak formulations of both domains $\alpha=a, p$.

The normal derivative of the pressure vanishes on the rigid surfaces $\Gamma_{i n c}$ and $\Gamma_{w}$. Equation (2) implies that

$$
\frac{\partial \hat{p}_{\alpha}}{\partial n}+\mathrm{i} k_{B}(\boldsymbol{\kappa} \cdot \mathbf{n}) \hat{p}_{\alpha}=0, \quad \text { for } \mathbf{x} \in\left(\Gamma_{i n c} \cup \Gamma_{w}\right),
$$

thus the boundary terms vanish on these surfaces. The boundary term pairs on the lateral boundaries also vanish because of the periodicity of $\hat{p}_{\alpha}$ and $\hat{q}_{\alpha}$ and due to the fact that the normal vectors point in opposite directions. The normal velocity continuity Eq. (2) on $\Gamma_{c}$ is automatically accounted for by removing the boundary integral.

The resolution is generally performed by fixing $k_{B}$ (real) with $\omega$ the eigenvalue. Propagating modes correspond to real frequencies whereas evanescent modes (i.e., band gap) correspond to complex frequencies. However, all the coefficients in porous medium are frequency dependent, which makes the eigenvalue problem highly non-linear in $\omega$. Therefore, it is preferable to solve the 
quadratic eigenvalue problem at fixed $\omega$ with $k_{B}$ as eigenvalue $^{37,38}$.

Once the terms involving the power of $k_{B}$ have been collected, we get the following quadratic eigenvalue problem expressed in $k_{B}$,

$$
D\left(k_{B}\right) \equiv K_{0}+K_{1} k_{B}+K_{2} k_{B}^{2}=0 .
$$

The operators $K_{i}(i=0, \ldots, 2)$ are combinations of bilinear operators involving the periodic test function and the periodic pressure field. Their definitions are given in appendix B. Due to the fact that the porous material parameters are complex, the operators $K_{i}(i=0, \ldots, 2)$ are not hermitian but simply complex symmetric or complex skew symmetric ${ }^{39}$. With or without inclusion, this kind of system is closely related with the existence of nonhermitian degeneracies ${ }^{31}$ called exceptional points ${ }^{28-30}$ (EPs). At each EP, both the eigenvalues and the right eigenvectors of two modes coalesce at a branch point in the parameters plane. The parameters can be a material property, such as density, dimension, position or the frequency etc. The EPs are different from the degeneracies encountered for hermitian operators where only the eigenvalues coalesce, while the corresponding eigenvectors are still orthogonal (e. g., classical geometrical symmetry degeneracies). One example about the existence of EP appears in duct acoustics with impedance boundary condition $^{33}$. When the impedance (parameter) is tuned to the Cremer's optimum impedance ${ }^{25-27}$, this leads to the highest modal attenuation. This degeneracy has also been observed in computational acoustic provided that the normalization of the lined duct modes with respect to the bi-orthogonality relation fails ${ }^{40}$. Lawrie et al. ${ }^{9,41}$ have related this norm to the derivative of the dispersion relation which vanishes for double roots.

\section{Implementation details}

The meshes are performed with $\mathrm{Gmsh}^{42}$. The computations are carried out using Lagrange quadratic finite elements with FreeFEM $++{ }^{43} \mathrm{FE}$ implementation. The first twenty eigenvalues of smallest magnitudes are solved with Two-level Orthogonal Arnoldi (TOAR) algorithm dedicated to polynomial eigenvalue problem without explicitly creating matrices of the linearized problem and suitable for non hermitian problems. The implementation is provided by $\operatorname{SLEPc}^{44}$ (Scalable Library for Eigenvalue Problem Computations). The numerical method has been validated with analytical solution for a homogeneous waveguide (air/porous).

\section{EXPERIMENTAL APPROACH}

\section{A. Experimental setup}

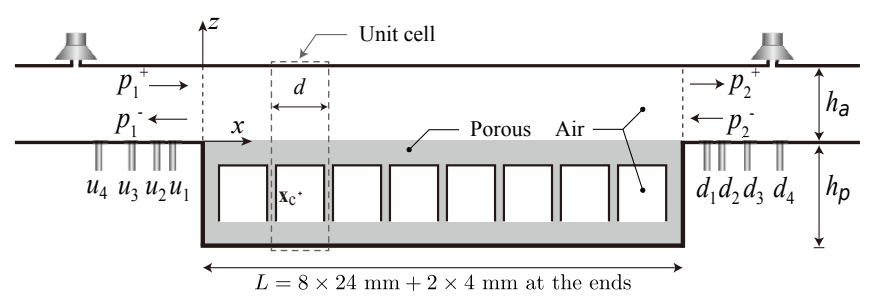

FIG. 2. A $2 \mathrm{D}$ schematic view of the experimental setup. The measured sample, of length $L=200 \mathrm{~mm}$, contains 8 unit cells. $\mathbf{x}_{c}$ is the center of the rigid inclusion.

The test facility ${ }^{45}$ used in this study is schematically depicted in Fig. 2. A two sources method is used: the acoustic waves are produced by two loudspeakers and propagate in a rectangular duct (width along $y$ direction $W=100 \mathrm{~mm}$, height $h_{a}=15 \mathrm{~mm}$ ). Two anechoic terminations are used to avoid resonant conditions in the duct. Note that the test material is put in a big rigid cavity.

The acquisition of signals is performed by Agilent VXI 1432 hardware platform which drives the source excitation synchronously with the acoustic pressure signals recording. A swept-sine over the frequency range 30$3500 \mathrm{~Hz}$ is used with a frequency increment of $10 \mathrm{~Hz}$.

Two series of four microphones (B\&K 4938 1/4", preamplifier B\&K 2670 with Nexus) mounted in the upstream $\left(u_{i}, i=1, \ldots, 4\right)$ and downstream $\left(d_{i}\right)$ of the test material section are used to measure the acoustical pressure. The use of $2 \times 4$ microphones allows an overdetermination of the transmitted and reflected waves on both sides of the test material and avoids the problems in the precision of measurement when the acoustic wavelength is close to half the distance between two microphones. Those microphones are located at the positions: $x_{u 1}-x_{u 2}=x_{d 1}-x_{d 2}=63.5 \mathrm{~mm}, x_{u 1}-x_{u 3}=x_{d 1}-x_{d 3}=$ $211.5 \mathrm{~mm}$, and $x_{u 1}-x_{u 4}=x_{d 1}-x_{d 4}=700 \mathrm{~mm}$.

In the frequency range $0-3400 \mathrm{~Hz}$, only 2 acoustic modes can propagate in the rigid ducts: the plane wave and the first-order mode along dimension $W$. The microphones are located just at the center of dimension $W$, which means that the second mode is not measured and only the plane waves is captured.

\section{B. Measuring technique}

The aim of the experimental apparatus is to measure the transfer matrix or the scattering matrix of a test material. The scattering matrix for the plane wave relates the scattered pressure amplitudes $p_{2}^{+}$and $p_{1}^{-}$(see Fig. 2) 
to the incident pressure amplitudes $p_{1}^{+}$and $p_{2}^{-}$by

$$
\left(\begin{array}{c}
p_{1}^{-} \\
p_{2}^{+}
\end{array}\right)=\left[\begin{array}{ll}
R^{+} & T^{-} \\
T^{+} & R^{-}
\end{array}\right]\left(\begin{array}{c}
p_{1}^{+} \\
p_{2}^{-}
\end{array}\right)=\mathrm{S}\left(\begin{array}{c}
p_{1}^{+} \\
p_{2}^{-}
\end{array}\right),
$$

where $T^{+}$and $T^{-}$are the anechoic transmission coefficients, $R^{+}$and $R^{-}$are the anechoic reflection coefficients, and superscripts \pm indicate the direction of wave propagation along the $x$ axis, and the subscripts ' 1,2 ' indicate the upstream and downstream of the duct. The method of measurement used in the present study is called "the 2 sources method". Two measurements are made in two different states of the system. These different states are obtained by switching on the upstream source, the downstream source being switched off (measurement I), and vice versa (measurement II) (see Ref. 45 for details).

\section{Description of the configurations}

The porous material is a metallic foam (RECEMAT, NC4753.05 nickel-chromium alloy) that has been chosen to avoid any skeleton vibrations. It is supplied as plate of thickness $5 \mathrm{~mm}$, and machined to the setup dimension. One layer of this metallic foam plate will be labelled by "P" in the following. The JCA parameters of the metallic foam used in the fluid equivalent model have been measured on another setup. The values are porosity $\phi=0.99$, tortuosity $\alpha_{\infty}=1.17$, viscous characteristic length $\Lambda=1 \times 10^{-4} \mathrm{~m}$, thermal characteristic length $\Lambda^{\prime}=2.4 \times 10^{-4} \mathrm{~m}$, and air flow resistivity $\sigma=6.9 \times 10^{3}$ $\mathrm{N} \mathrm{m}^{-4}$ s. Five layers "P" of this porous material are assembled together to form a $25 \mathrm{~mm}$ thick uniform porous material, labeled by " $5 \mathrm{P}$ " in the following. This configuration represents a reference homogeneous porous liner.

The inclusions are metallic hollow cylinders with one closed end and one open end, as illustrated in Fig. 3(a) and embedded in the porous material as in Fig. 3(c). The cylinder we considered in this work is with external diameter $22 \mathrm{~mm}$, wall thickness $0.5 \mathrm{~mm}$, bottom thickness $2 \mathrm{~mm}$, and the total height $15 \mathrm{~mm}$, as shown in Fig. 3(a). Here $\mathbf{x}_{c}$ is the center of the cylinder and indicates the inclusion position in the unit cell. Configurations with two different inclusion orientations are investigated. If the closed ends of all the cylinders are on the bottom and direct towards the rigid backing, it's labeled by "ப"; inversely, " $\sqcap$ " when all the closed ends are on the top and direct towards the duct (see e.g. a 2D view in Fig. 2). In order to embed the inclusions in the porous material, holes are drilled in three metallic foam layers, as we can see from Figs. 3(b) and (c). To keep the same height on all the configurations, one layer of metallic foam " $\mathrm{P}$ " is added both on the top and bottom of the inclusions, leading to the configurations $\mathrm{P}-\sqcap-\mathrm{P}$ and $\mathrm{P}-\sqcup-\mathrm{P}$ mentioned in the following.

Acoustic measurements have been performed with cylinders embedded in the center of the cells $\mathbf{x}_{c}=$ $\left(0,0,-h_{p} / 2\right)$ (see in Fig. 2).
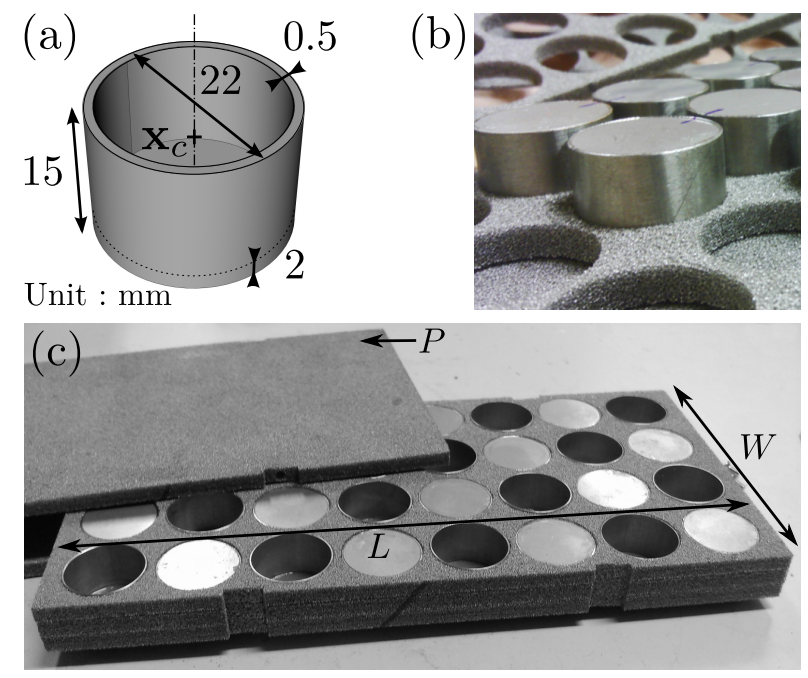

FIG. 3. Pictures of (a) an open cylinder inclusion (filled with air), (b) zoom of the drilled metallic foam layers of height 5 $\mathrm{mm}$, and (c) a whole sample with cylinders (Fig. 3(a)) embedded in an alternated way.

\section{RESULTS AND DISCUSSION}

\section{A. Comparisons between the model and the measurements}

The measured scattering matrix coefficients are given in Fig. 4. Comparisons between the measured Transmission Loss TL $=-20 \log |T|$ for 8 unit cells and FE Bloch mode attenuation $^{25}$ for a length $L$

$$
\operatorname{att}\left(k_{B}\right)=8.68 \cdot \operatorname{Im} k_{B} L,
$$

are shown in Fig. 5.

The oscillations in the reflection coefficient in Fig. 4(b) are linked to the wave reflection at the ends of the material (see Fig. 2). The reflection coefficient modulus oscillates around $|R|=0.45$ which is close to the approximation $(1-r) /(1+r)=0.45$, valid at low frequencies for an expansion with the height ratio $r=h_{a} /\left(h_{a}+h_{p}\right)$ and without porous material ${ }^{13}$. The first frequencies where the reflection coefficient is minimum $\left(f_{n} \approx n c_{a} /(2 L), n\right.$ integer) can also be estimated with an empty expansion chamber model ${ }^{46}$.

These oscillations, due to the interface discontinuities, can also be observed in the transmission coefficient (see Fig. 4(a) ) and in the measured TL (see Fig. 5). They can also explain the differences between the measured TL and Bloch mode attenuation in Fig. 5. The latter generally has a smaller attenuation value because the reflected waves at discontinuities send back the sound intensity to upstream. Nonetheless, the main trends are perfectly recovered with the least attenuated Bloch mode, especially for the attenuation peak frequency observed in Fig. 5(c).

From Fig. 4, the transmission coefficient or more conveniently the measured TL, reveals that below $500 \mathrm{~Hz}$ 

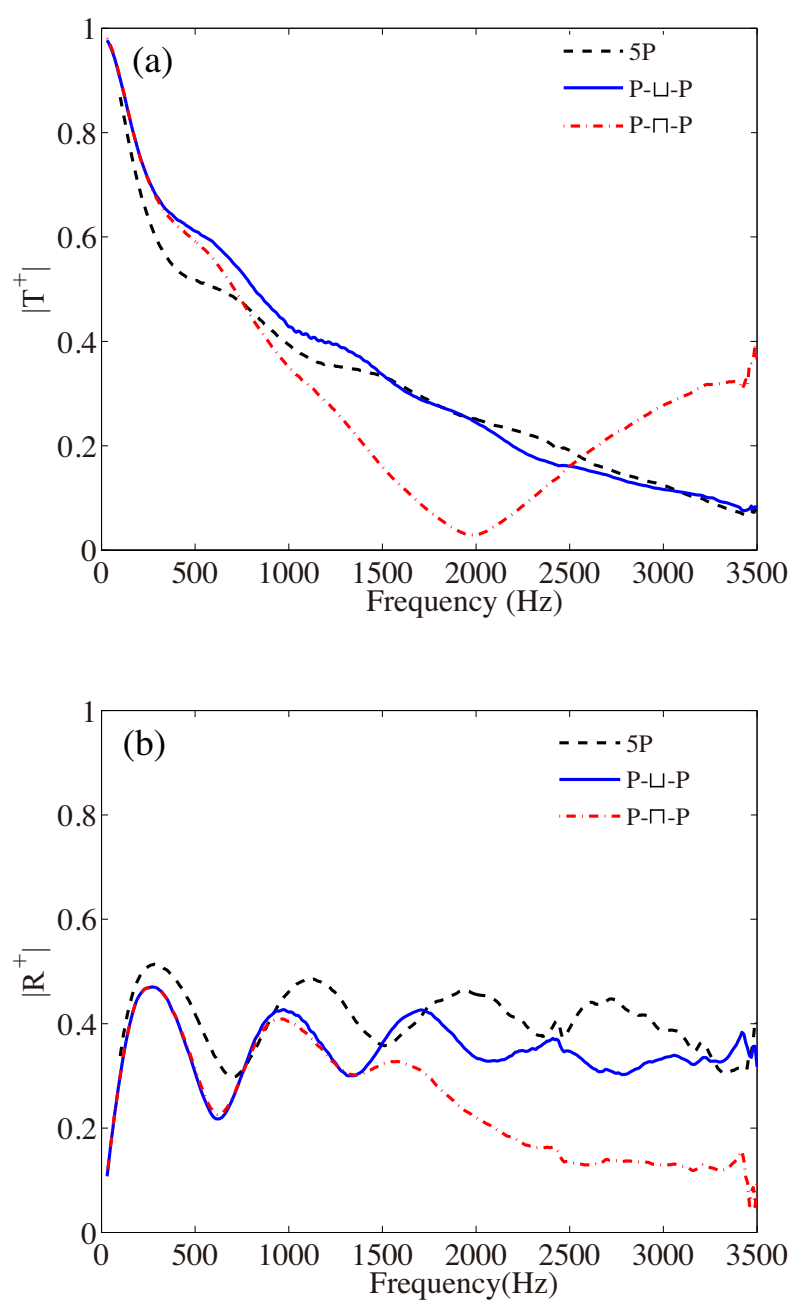

FIG. 4. Measured scattering matrix coefficients for the three

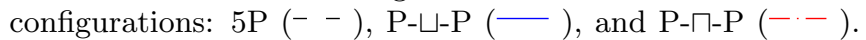
(a) Absolute value of transmission coefficient $T^{+}$and (b) absolute value of reflexion coefficient $R^{+}$.

neither the tested inclusion shape nor its orientation are important because the pressure in the liner is nearly constant for the first mode. The TL depends mainly on the porous material volume, which is bigger for the configuration $5 \mathrm{P}$, due to the air enclosed in the cylinders.

Above $500 \mathrm{~Hz}$, the inclusion orientation starts to become significant. The measured TL of configuration P-ப$\mathrm{P}$ is quite similar to the one of $5 \mathrm{P}$. This is also true for the Bloch mode attenuation curves, as shown in Figs. 5(a) and (b). When the cylinders are reversed, i.e., for configuration $\mathrm{P}-\sqcap-\mathrm{P}$, a TL peak can be observed, see Fig. 5(c). As TL is driven by the least attenuated mode, the peak appears when the first and the second mode attenuation curves cross. The frequency of the TL peak is closely related to the effective height $h_{p}^{\prime}$, illustrated in the inserts of Figs. 5(b) and (c), respectively and which corresponds to an estimation of the longest tortuous path within the liner. With the presence of the inclusion, $h_{p}^{\prime}>h_{p}$ and the liner behaves like a thicker one. When the cylinder is in the $\sqcap$ orientation, the effective height $h_{p}^{\prime}$ appears to be larger than when the cylinder is in the $\sqcup$ orientation and the second mode becomes nearly cut-on at lower frequency. Similar effects have also been observed in Ref. 18 for a $2 \mathrm{D}$ absorption problem.

\section{B. Exceptional points}

The crossing of the two Bloch mode attenuations observed in Fig. 5(c) is closely related to the existence of an EP. When periodic inclusions are embedded, new parameters can be used to tune the EP, for example the shape, the size or the position of the inclusion.

To illustrate the existence of the EP in the parameter plane of frequency and inclusion position, the cylinder center $\mathbf{x}_{c}$ is moved above ( $\delta$ is positive) or below ( $\delta$ is negative) the center of the unit cell, as illustrated in the insert of Fig. 6. The effect on the Bloch mode attenuations is then investigated numerically.

By changing $\delta$, the inclusion behaves as a quarter wavelength resonator or as a Helmholtz resonator, when the distance between the inclusion and the rigid wall becomes small. This parametric study has been performed on weak contrasted waveguides, when $h_{p} \approx h_{a}$, corresponding to experimental setup, and on strong contrast waveguide when $h_{p} \ll h_{a}$ corresponding more to practical situations. The latter configuration makes also the dispersion curves interpretation easier.

\section{Weak contrast $h_{p} \approx h_{a}$}

Modal attenuations for the lower two Bloch modes as a function of frequency at different inclusion positions are given in Fig. 6. We can see that crossing (e.g. $\delta=2$ ) and avoided crossing (e.g. $\delta=-4.7)$ for the two mode attenuations are observed. With moving the cylinder center $\mathbf{x}_{c}$ below the center of the unit cell, the amplitude of the mode attenuations is getting larger until the appearance of an avoided crossing, e.g., the amplitude is smaller at $\delta=-4.70 \mathrm{~mm}$ than at $\delta=-4.61 \mathrm{~mm}$. This means that there exists a critical value of $\delta^{*}$ where the maximal modal attenuation for the least attenuated mode can be achieved, leading us to the optimization of the acoustic liner. The enhancement may be significant since for the nominal value $\delta=0$ the computed attenuation at about $2 \mathrm{kHz}$ is around $20 \mathrm{~dB}$ (see Fig. 5(c)) and for the optimal value $\delta^{*}$ the attenuation is around $40 \mathrm{~dB}$. This maximal value corresponds to a degenerated case where the lower two Bloch modes coalesce to form a double root.

Double root of the dispersion equation has already been observed for a waveguide lined with locally reacting liner. It has been shown by Tester ${ }^{25}$ that Cremer's optimum impedance ${ }^{27}$ corresponds to a double root of the eigenvalue problem and is an EP in complex impedance (admittance) plane ${ }^{33}$. 

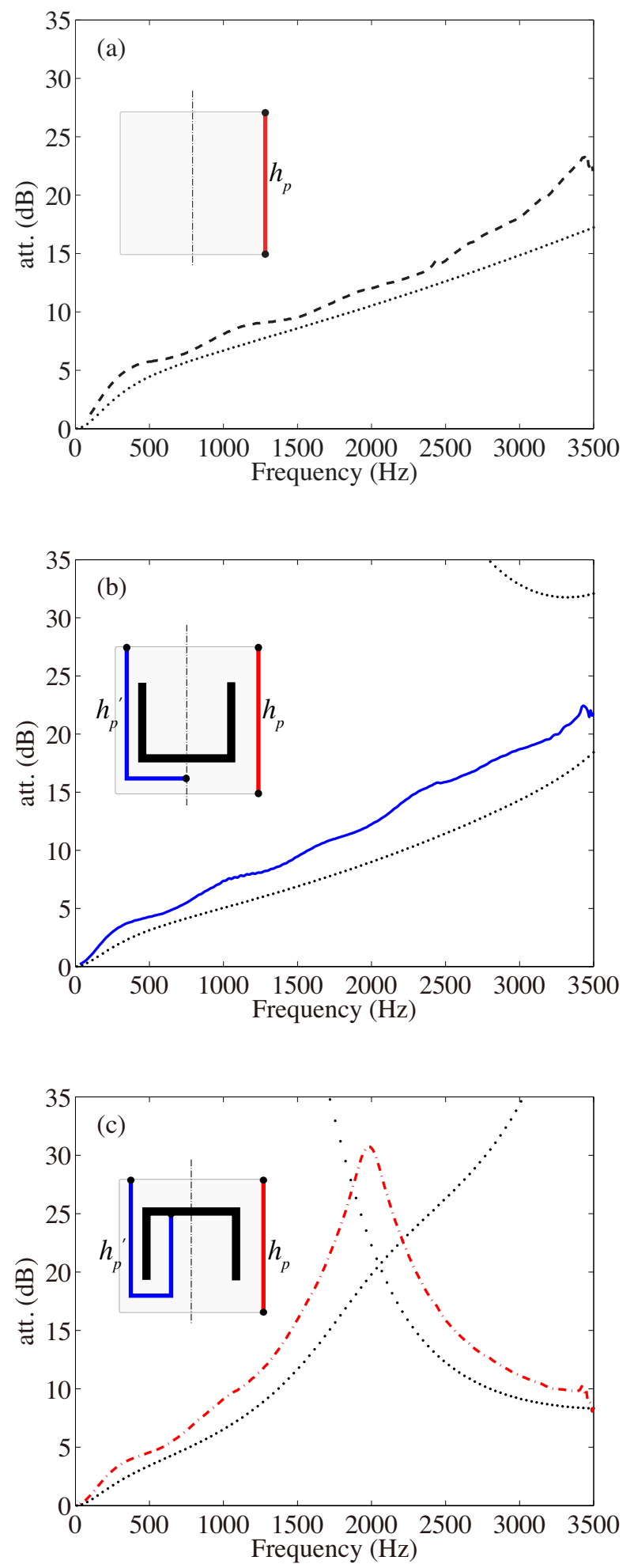

FIG. 5. Comparisons between the Bloch mode attenuation computed using Eq. (9) (..) and the measured transmission loss $(--,-\ldots,--$, respectively) with 8 unit cells for the configurations: (a) 5P, (b) P-ப-P, and (c) P-П-P. The inserted schemes in (b) and (c) show the effective heights $h_{p}^{\prime}$ of the latter two configurations.

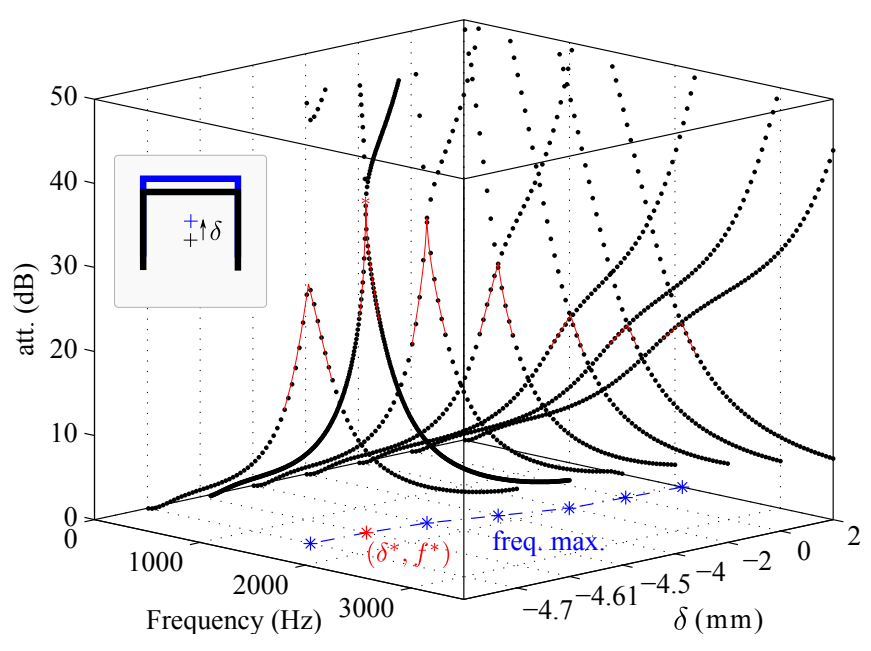

FIG. 6. Mode attenuations for the lower two Bloch modes as a function of frequency at different inclusion positions $\delta$. $\delta$ is positive when the cylinder centre $\mathbf{x}_{c}$ is moved above the center of the cell, and negative when $\mathbf{x}_{c}$ is moved below the center of the cell.

To illustrate the existence of the EP near the (avoided) crossing in Fig. 6 and the importance of its role on the sound attenuation optimization, we plot in Fig. 7 the real and imaginary parts of the lower two Bloch wavenumbers $k_{B}$ as a function of frequency at two different inclusion positions $\delta=-4.63$ and $\delta=-4.61 \mathrm{~mm}$. When $\delta=-4.63 \mathrm{~mm}$, there is a crossing for Re $k_{B}$ and an avoided crossing for $\operatorname{Im} k_{B}$ (see "+" in Fig. 7 (a) and (b)), while for $\delta=-4.61 \mathrm{~mm}$, there is an avoided crossing for $\operatorname{Re} k_{B}$ and a crossing for $\operatorname{Im} k_{B}$ (see " $\times$ " in Fig. 7 (a) and (b)). The type change of the avoided crossings shows that there exists a critical value $\delta^{*}$, for which the two wavenumbers $k_{B}$ will cross at a critical value of frequency $f^{* 35}$. Thus, $\left(\delta^{*}, f^{*}\right) \sim(-4.62 \mathrm{~mm}, 1570 \mathrm{~Hz})$ is called an EP in the parameter plane of $(\delta, f)$, where the lower two Bloch modes in Fig. 7 will coalesce. This EP leads to the maximal sound attenuation for the least attenuated mode, as it can be observed in Fig. 6 .

Nonetheless, this interpretation must be tempered. Indeed, when two modes tend to merge into a single one, a typical linear growth of the pressure field with respect to the axial coordinate occurs: the underlying beating phenomenon can be understood as the sum of two waves with infinitely close wavenumber. The EP will lead to the optimal sound attenuation if the linear growth remains negligible with respect to the exponential modal attenuation. For a finite liner, the global efficiency will be also strongly conditioned by the matching efficiency at the inlet/outlet of the silencer.

Using a Taylor expansion as in Refs. 25 and 26 in the vicinity of the double root $k_{B}^{*}$ and assuming that there is no higher multiplicity root, the dispersion equation can 

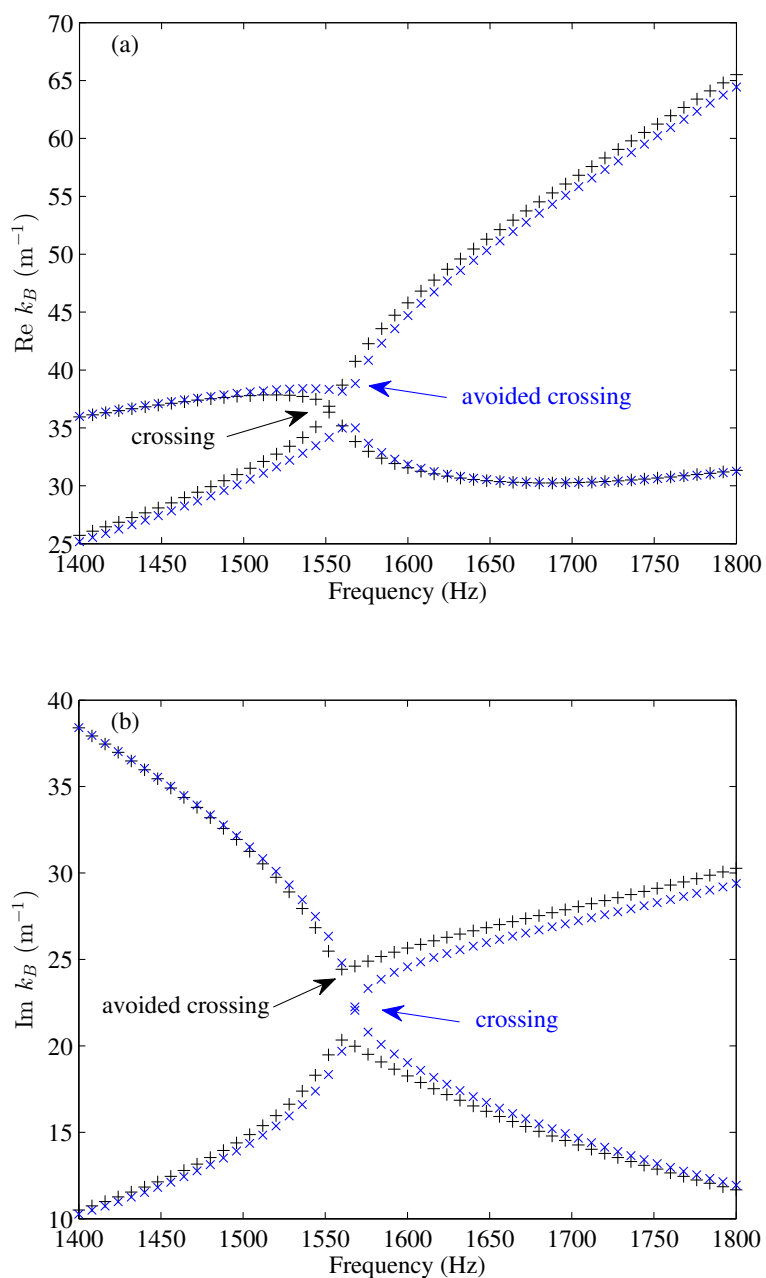

FIG. 7. Crossing and avoided crossing of (a) real and (b) imaginary parts of the lower two Bloch wavenumbers when $h_{a}=15 \mathrm{~mm}$ with "+" for $\delta=-4.63 \mathrm{~mm}$ and " $\times$ " for $\delta=$ $-4.61 \mathrm{~mm}$

be approached by

$$
\begin{aligned}
D\left(k_{B}, \delta, f\right) \approx D^{*} & +\left(k_{B}-k_{B}^{*}\right) \frac{\partial D^{*}}{\partial k_{B}}+\frac{1}{2}\left(k_{B}-k_{B}^{*}\right)^{2} \frac{\partial^{2} D^{*}}{\partial k_{B}^{2}} \\
& +\left(\delta-\delta^{*}\right) \frac{\partial D^{*}}{\partial \delta}+\left(f-f^{*}\right) \frac{\partial D^{*}}{\partial f}
\end{aligned}
$$

where $D^{*} \equiv D\left(k_{B}^{*}, \delta^{*}, f^{*}\right)$ for the sake of conciseness. On the modal branches, $D\left(k_{B}, \delta, f\right)$ vanishes. Recalling that $k_{B}^{*}$ is a double root of the dispersion equation, $D^{*}$ and $\frac{\partial D *}{\partial k_{B}}$ also vanish, Eq. (10) leads to

$$
\begin{array}{r}
k_{B}-k_{B}^{*} \approx \pm\left[( - \frac { 1 } { 2 } \frac { \partial ^ { 2 } D ^ { * } } { \partial k _ { B } ^ { 2 } } ) ^ { - 1 } \left(\left(\delta-\delta^{*}\right) \frac{\partial D^{*}}{\partial \delta}+\right.\right. \\
\left.\left.\left(f-f^{*}\right) \frac{\partial D^{*}}{\partial f}\right)\right]^{1 / 2} .
\end{array}
$$

Each mode corresponds to different Riemann sheets of

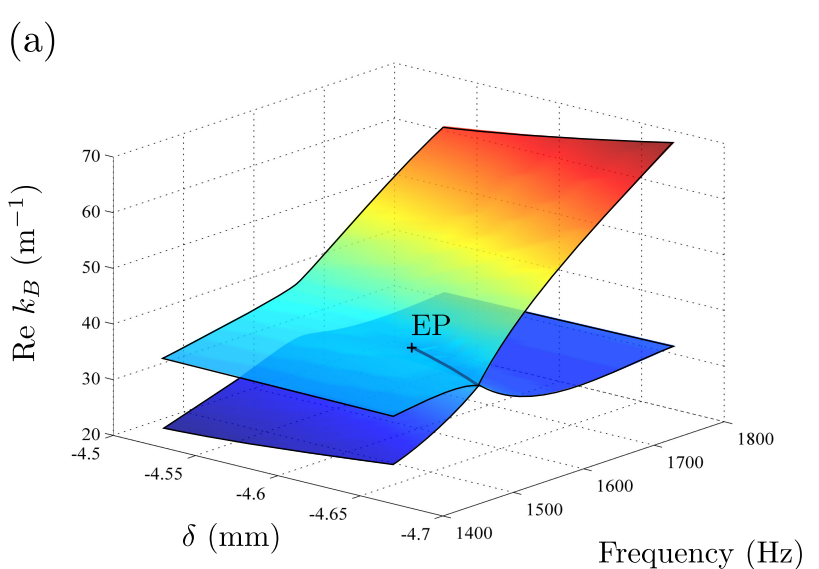

(b)

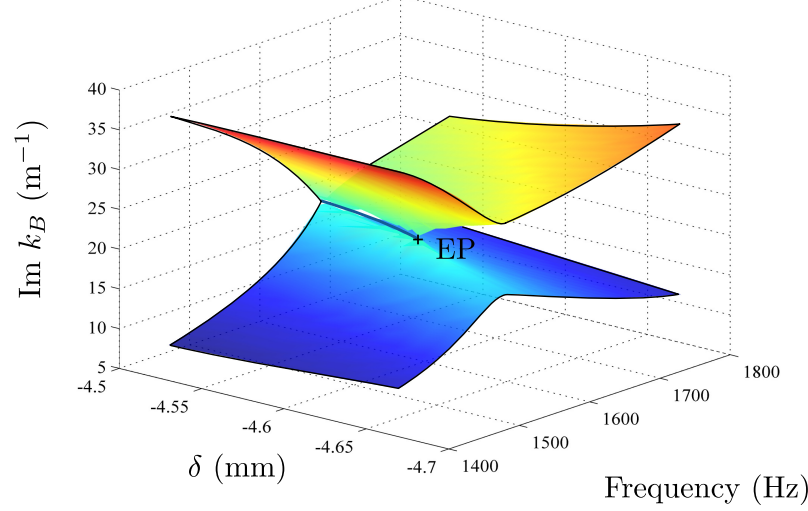

FIG. 8. Riemann sheet of the real (a) and imaginary (b) part of the lower attenuated Bloch wavenumber. The line indicates the crossing of the different sheets.

wavenumber and the two modes are connected by a square root branch point ${ }^{25,26,29}$ in the $(\delta, f)$-parametric space (here the parameters are real) as shown in Fig. 8. The branch point singularity is responsible for the crossing or the avoided crossing depending on the followed path in the $(\delta, f)$-parametric space.

\section{Strong constrast $h_{p} \ll h_{a}$}

In this section, all dimensions remain unchanged except that the airway height is set as $h_{a}=135 \mathrm{~mm}$. As in the previous case the existence of an EP can be exhibited in the $(\delta, f)$ parametric space for the least attenuated mode. In Fig. 9, the real and imaginary parts of the lower two Bloch wavenumbers $k_{B}$ are plotted as a function of frequency for two different inclusion positions $\delta=-4.0$ and $\delta=-3.9 \mathrm{~mm}$. When $\delta=-4.0 \mathrm{~mm}$, there is a crossing for $\operatorname{Re} k_{B}$ and an avoided crossing for $\operatorname{Im} k_{B}$ (see "+" in Fig. 9 (a) and (b)), while for $\delta=-3.9 \mathrm{~mm}$, there is an avoided crossing for Re $k_{B}$ and a crossing for $\operatorname{Im} k_{B}$ (see " $\times$ " in Fig. 9 (a) and (b)). The type change of the avoided crossings shows that there exists an EP at $\left(\delta^{*}, f^{*}\right) \sim(-4 \mathrm{~mm}, 1380 \mathrm{~Hz})$. Comparing to the different 
value of EP $(-4.62 \mathrm{~mm}, 1570 \mathrm{~Hz})$ obtained in the previous section when $h_{p} \approx h_{a}$, it can be noted that the EP $\left(\delta^{*}, f^{*}\right)$ depends on the ratio between $h_{p}$ and $h_{a}$.

The complete dispersion curves for this configuration are given in Fig. 10. For the higher order modes, the coupling between the two waveguides becomes smaller and the present configuration is comparable to the limit cases of uncoupled waveguides. Hence, when $h_{p} \ll h_{a}$, it becomes easier to analyze the eigenvectors behavior in the presence of an EP.

At low frequency, the first mode labeled $m_{0}$ couples strongly the air and porous domains and the pressure is nearly constant across the height. Moreover, the dispersion curves of the higher order modes in the airway can be well approximated by rigid wall boundary condition on $\Gamma_{c}$ (see the - curves in Fig. 10). In the meantime, a mode localized in the liner can be seen. It can be well approximated by assuming $p_{p}=0$ boundary condition on the coupling interface $\Gamma_{c}$ and solving the problem (7) in the liner alone (see $m_{L}$ with + markers in Fig. 10). The Dirichlet boundary condition is quite natural because the characteristic impedance of the porous material in diffusive regime tends to infinity $\propto \sqrt{\sigma / \omega}$ as the frequency goes to 0 (see appendix A). The rough approximation of the $1 \mathrm{D}$ reflection coefficient at the interface $\Gamma_{c}$ is then 1 from the airway to the porous, and -1 inversely. This explains that the modal behavior of the coupled system is close to the Neumann-Neumann problem in the airway and to the Dirichlet-Neumann problem in the liner respectively.

When the frequency increases, this asymptotic behavior is no longer valid. In the vicinity of the EP, the coupling between the two waveguides is strong. The system is also very dispersive and the Bloch wavenumbers are very sensitive to small frequency shift. The eigenvalues of the modes labeled $m_{1}$ and $m_{0}$ merge. As mentioned previously, it can be seen that the two eigenvectors also merge and the pressure field is localized in the liner. The more the pressure field is localized in the porous material, the more the attenuation increases. Close to the $\mathrm{EP}$, the two lower attenuated modes are confined in the liner, hence it explains why the modal attenuation is the highest.

After the EP, the two modes become again different. For one of them, labeled $m_{S}$, the pressure field remains localized inside the liner and this mode becomes more and more attenuated. At higher frequency (keeping $k_{B} \ll \pi / d$ ) the mode behavior is well approximated by the Dirichlet-Neumann mode $m_{L}$ in the liner alone. Once projected on the frequency-Re $k_{B}$ plane, the EP occurs at a frequency near the crossing between the first mode of the rigid duct and the $m_{L}$ mode. It can be noticed that similar qualitative results are obtained by using air characteristic impedance $\rho_{a} c_{a}$ boundary condition or Dirichlet boundary condition on $\Gamma_{c}$.

It is noteworthy that the group velocity of $m_{S}$ is smaller comparing to the sound speed in homogeneous the porous material $c_{p}$. It can be explained by the tor-
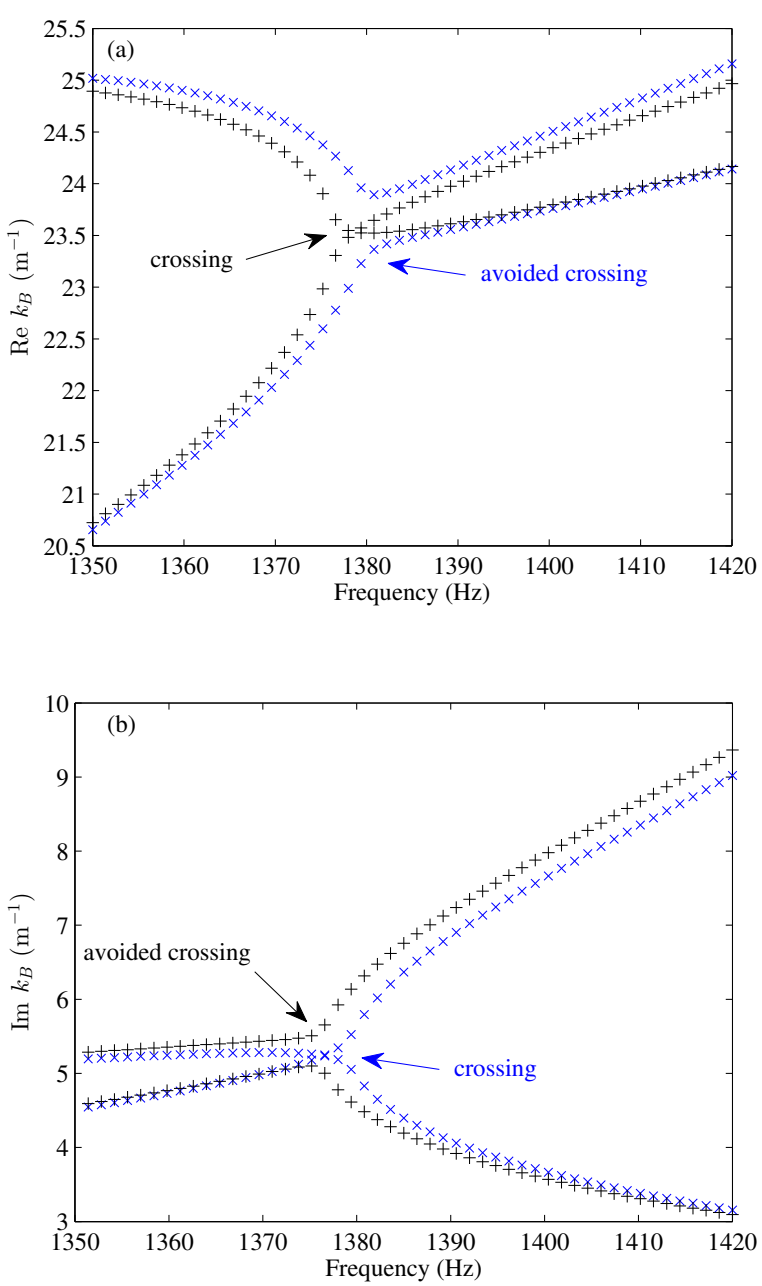

FIG. 9. Crossing and avoided crossing of (a) real and (b) imaginary parts of the lower two Bloch wavenumbers when $h_{a}=135 \mathrm{~mm}$ with "+" for $\delta=-4.0 \mathrm{~mm}$ and " $\times$ " for $\delta=$ $-3.9 \mathrm{~mm}$

tuous path of the wave in the liner due to the inclusion.

The localized mode inside the liner is equivalent to a quasi-surface wave along the impedance wall as observed theoretically by Bi and Pagneux in Ref. 34. They use an analogy with resonance trapping phenomenon in open quantum system to shed the light on the EP and waveguide mode behaviors.

For the other mode, above the EP frequency, the eigenvector is becoming similar to the mode $m_{1}$ (1 nodal line). This mode is labeled $m_{1}^{\prime}$ and everything happens as if the quasi constant mode $m_{0}$ has switched with the first higher mode $m_{1}$ at low frequency, as shown in Fig. 10. The group velocity tends to $c_{a}$ when the frequency increases, indicating that this mode becomes a perturbation of a rigid duct mode. 

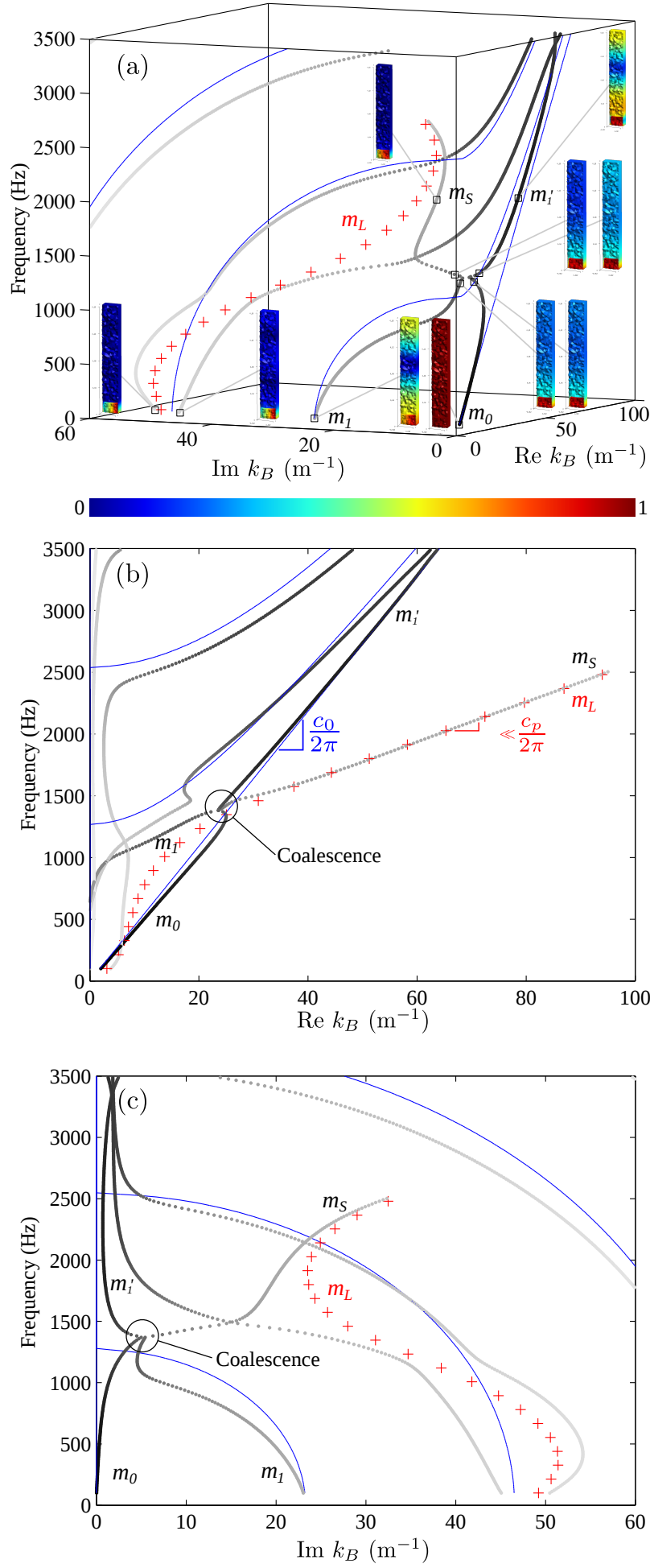

FIG. 10. Dispersion curves for the Bloch wavenumber when $h_{a}=135 \mathrm{~mm}$ and $\delta=-4.0 \mathrm{~mm}$ a) $3 \mathrm{D}$ view, b) projection in the frequency-Re $k_{B}$ plane, c) projection in the frequency$\operatorname{Im} k_{B}$ plane. The markers $\cdot$ present the numerical solutions for the air duct with metaporous liner, the lines - correspond to modes in a rigid acoustic waveguide of height 135 $\mathrm{mm}$ and the markers + represent the solution for the liner alone (with Dirichlet b.c. on top). The inserted pictures show the $(O, x, z)$-cut of the modulus of the right eigenvectors at $100,1375,1385$ and $2000 \mathrm{~Hz}$. The eigenvectors are normalized such the maximum value is red $(=1)$ and blue is 0 .

\section{CONCLUSIONS}

In this paper porous material with embedded periodic rigid resonant inclusions has been investigated experimentally and numerically to enhance the sound attenuation at low frequency for acoustic ducts. Good agreements have been observed between the measurements and the predicted results. The measured transmission loss peak observed in the partially lined acoustic duct can be explained by the crossing (or avoided crossing) of the lower two Bloch mode attenuations in the infinite periodic waveguide.

It has been shown that the embedded inclusions can greatly enhance the sound attenuation in a partially lined duct if the inclusion shape and position are carefully chosen. Due to the existence of the exceptional point (EP) in the parameter space of the inclusion position and frequency, different types of crossing and avoided crossing are observed for the Bloch wavenumber. At the EP, where both the eigenvalues and the eigenvectors of the lower two Bloch modes coalesce, a maximal sound attenuation can be achieved because of a localized mode in the liner.

This paper opens the way to the understanding of the design of metaporous materials or metamaterials for waveguide attenuation. The inclusion position can be easily used to tune the material and to get close to an EP. The EP based design generalizes to non locally reacting material the concept of optimal impedance ${ }^{25-27,33}$ developed initially by Cremer and Tester. Work is on going to identify the conditions leading to EP and its efficiency on finite length configuration as it has been done for impedance boundary condition ${ }^{2,3,33}$.

\section{ACKNOWLEDGMENT}

The authors acknowledge the support from the European Union through ITN-project FlowAirS (Contract No. FP7-PEOPLE-2011-ITN-289352) and the support from the French Research National Agency and the Research Foundation for Aeronautics and Space through the project Metaudible (ANR-13-BS09-0003-01).

\section{Appendix A: Rigid frame equivalent model}

Porous materials with rigid skeleton (and quite regular pore shape), such as the porous material involved in this study, are well described by the Johnson-ChampouxAllard ${ }^{15}$ (Chap. 5) equivalent fluid model. This equivalent fluid has the equivalent density $\left(e^{-i \omega t}\right)$.

$$
\rho_{p}=\frac{\alpha_{\infty} \rho_{a}}{\phi}\left[1+\mathrm{i} \frac{\sigma \phi}{\omega \rho_{a} \alpha_{\infty}} G_{J}(\omega)\right],
$$


and the bulk modulus,

$$
K_{p}=\frac{\gamma P_{a} / \phi}{\gamma-(\gamma-1)\left[1+\mathrm{i} \frac{8 \eta}{\Lambda^{\prime 2} \operatorname{Pr} \omega \rho_{a}}\left(1-\mathrm{i} \rho_{a} \frac{\omega \operatorname{Pr} \Lambda^{\prime 2}}{16 \eta}\right)^{1 / 2}\right]^{-1}} .
$$

Here, $G_{J}(\omega)=\sqrt{1-\frac{4 \mathrm{i} \alpha_{\infty}^{2} \eta \rho_{a} \omega}{\sigma^{2} \Lambda^{2} \phi^{2}}}, \phi$ is the porosity, $\sigma$ is the flow resistivity, $\Lambda$ is the vicious length, $\Lambda^{\prime}$ is the thermal length, $\alpha_{\infty}$ is the tortuosity. Moreover, $\gamma$ is the air specific heat ratio and $P_{a}$ is the atmospheric pressure, $\operatorname{Pr}$ is the Prandtl number and $\eta$ is the dynamic viscosity. It is important to note, the viscosity is taken into account in the porous material pore but is neglected in the surrounding fluid.

If $\omega \ll \omega_{b}=\frac{\sigma \phi}{\rho_{a} \alpha_{\infty}}$, viscous forces are dominant and the effective parameters can be replaced by the low-frequency approximations at first order ${ }^{12}$

$$
\rho_{p} \approx \mathrm{i} \rho_{a} \frac{\alpha_{\infty}}{\phi} \frac{\omega_{b}}{\omega}
$$

and

$$
K_{p} \approx \frac{P_{a}}{\phi}
$$

\section{Appendix B: Operators details}

Here, are detailed the bilinear operators listed in Eq. (7) arising from Eq. (5):

$$
\begin{aligned}
K_{0} & =\frac{1}{\rho_{a}} \times\left[-\int_{\Omega_{a}} \nabla \hat{q}_{a} \cdot \nabla \hat{p}_{a} \mathrm{~d} \Omega+k_{a}^{2} \int_{\Omega_{a}} \hat{q}_{a} \hat{p}_{a} \mathrm{~d} \Omega\right] \\
& +\frac{1}{\rho_{p}} \times\left[-\int_{\Omega_{p}} \nabla \hat{q}_{p} \cdot \nabla \hat{p}_{p} \mathrm{~d} \Omega+k_{p}^{2} \int_{\Omega_{p}} \hat{q}_{p} \hat{p}_{p} \mathrm{~d} \Omega\right], \\
K_{1} & =\frac{\mathrm{i}}{\rho_{a}} \int_{\Omega_{a}}\left(-\nabla \hat{q}_{a} \cdot\left(\boldsymbol{\kappa} \hat{p}_{a}\right)+\left(\boldsymbol{\kappa} \hat{q}_{a}\right) \cdot \nabla \hat{p}_{a}\right) \mathrm{d} \Omega \\
& +\frac{\mathrm{i}}{\rho_{p}} \int_{\Omega_{p}}\left(-\nabla \hat{q}_{p} \cdot\left(\boldsymbol{\kappa} \hat{p}_{p}\right)+\left(\boldsymbol{\kappa} \hat{q}_{p}\right) \cdot \nabla \hat{p}_{p}\right) \mathrm{d} \Omega, \\
K_{2} & =-\left[\frac{1}{\rho_{a}} \int_{\Omega_{a}} \hat{p}_{a} \hat{q}_{a} \mathrm{~d} \Omega+\frac{1}{\rho_{p}} \int_{\Omega_{p}} \hat{p}_{p} \hat{q}_{p} \mathrm{~d} \Omega\right] .
\end{aligned}
$$

${ }^{1}$ H. H. Hubbard, Aeroacoustics of Flight Vehicles: Theory and Practice, Vol. 2 Noise Control, chapter 14, 1258, pp. 165205 (NASA Reference publication, Washington, United States) (1991).

${ }^{2}$ R. Kabral, L. Du, and M. Åbom, "Optimum sound attenuation in flow ducts based on the "exact" Cremer impedance", Acta Acust. united Ac. 102, 851-860 (2016).

${ }^{3}$ T. Bravo, C. Maury, and C. Pinhède, "Optimisation of microperforated cylindrical silencers in linear and nonlinear regimes", J. Sound Vib. 363, 359-379 (2016).

${ }^{4}$ D. Li, D. Chang, and B. Liu, "Enhancing the low frequency sound absorption of a perforated panel by parallel-arranged extended tubes", Applied Acoustics 102, 126-132 (2016).
${ }^{5}$ J.-P. Groby, W. Huang, A. Lardeau, and Y. Aurégan, "The use of slow waves to design simple sound absorbing materials", J. Appl. Phys. 117, 124903 (2015).

${ }^{6}$ Y. Aurégan and V. Pagneux, "Slow sound in lined flow ducts", J. Acoust. Soc. Am. 138, 605-613 (2015).

${ }^{7}$ Y. Aurégan, M. Farooqui, and J.-P. Groby, "Low frequency sound attenuation in a flow duct using a thin slow sound material", J. Acoust. Soc. Am. 139, EL149-EL153 (2016).

${ }^{8} \mathrm{~B}$. Nilsson and O. Brander, "The propagation of sound in cylindrical ducts with mean flow and bulk-reacting lining. I. modes in an infinite duct", J. Inst. Maths. Applics. 26, 269-298 (1980).

${ }^{9}$ J. B. Lawrie and R. Kirby, "Mode-matching without root-finding: Application to a dissipative silencer", J. Acoust. Soc. Am. 119, 2050-2061 (2006).

${ }^{10}$ B. Nennig, E. Perrey-Debain, and M. Ben Tahar, "A mode matching method for modelling dissipative silencers lined with poroelastic materials and containing mean flow", J. Acoust. Soc. Am. 128, 3308-3320 (2010).

${ }^{11}$ B. Nennig, M. Ben Tahar, and E. Perrey-Debain, "A displacement-pressure finite element formulation for analyzing the sound transmission in ducted shear flows with finite poroelastic lining", J. Acoust. Soc. Am. 130, 42-51 (2011).

${ }^{12}$ B. Nennig, R. Binois, E. Perrey-Debain, and N. Dauchez, "A homogenization method used to predict the performance of silencers containing parallel splitters", J. Acoust. Soc. Am. 137, 3221-3231 (2015).

${ }^{13}$ Y. Aurégan and D. Kumar Singh, "Experimental observation of a hydrodynamic mode in a flow duct with a porous material", J. Acoust. Soc. Am. 136, 567-572 (2014).

${ }^{14}$ R. J. Astley, "A comparative note on the effects of local versus bulk reaction models for air moving ducts lined on all sides", J. Sound Vib. 117, 191-197 (1987).

${ }^{15} \mathrm{~J} .-\mathrm{F}$. Allard, Propagation of Sound in Porous Media: Modeling Sound Absorbing Materials, 280pp (Chapman \& Hall, New York) (1993).

${ }^{16}$ O. Tanneau, J. B. Casimir, and P. Lamary, "Optimization of multilayered panels with poroelastic components for an acoustical transmission objective", J. Acoust. Soc. Am. 120, 1227-1238 (2006).

${ }^{17}$ J.-P. Groby, A. Duclos, O. Dazel, L. Boeckx, and W. Lauriks, "Enhancing absorption coefficient of a backed rigid frame porous layer by embedding circular periodic inclusions", J. Acoust. Soc. Am. 130, 3071-3780 (2011).

${ }^{18}$ B. Nennig, Y. Renou, J.-P. Groby, and Y. Aurégan, "A mode matching approach for modeling two dimensional porous grating with infinitely rigid or soft inclusions", J. Acoust. Soc. Am. 131, 3841-3852 (2012).

${ }^{19}$ J.-P. Groby, B. Nennig, C. Lagarrigue, B. Brouard, O. Dazel, and V. Tournat, "Enhancing the absorption properties of acoustic porous plates by periodically embedding Helmholtz resonators", J. Acoust. Soc. Am. 137, 273-280 (2015).

${ }^{20}$ C. Boutin and F.-X. Becot, "Theory and experiments on poroacoustics with inner resonators", Wave Motion 54, 76-99 (2015).

${ }^{21}$ J. Yang, J. S. Lee, and Y. Y. Kim, "Metaporous layer to overcome the thickness constraint for broadband sound absorption", J. Appl. Phys. 117, 174903 (2015).

${ }^{22}$ S. Griffiths, B. Nennig, and S. Job, "Porogranular materials composed of elastic Helmholtz resonators for acoustic wave absorption", J. Acoust. Soc. Am. 141, 254-264 (2017).

${ }^{23}$ J. Yang, J. S. Lee, and Y. Y. Kim, "Multiple slow waves in metaporous layers for broadband sound absorption", J. Phys. D: Appl. Phys. 50, 015301 (2017).

${ }^{24}$ V. Romero-Garcia, G. Theocharis, O. Richoux, A. Merkel, V. Tournat, and V. Pagneux, "Perfect and broadband acoustic absorption by critically coupled sub-wavelength resonators", Sci. Rep. 6, 19519 (2016).

${ }^{25} \mathrm{~B}$. J. Tester, "The optimization of modal sound attenuation in duct, in the absence of mean flow", J. Sound Vib. 27, 477-513 (1973). 
${ }^{26}$ E. L. Shenderov, "Helmholtz equation solutions corresponding to multiple roots of the dispersion equation for a waveguide with impedance walls", Acoust. Phys. 46, 357-363 (2000).

${ }^{27} \mathrm{~L}$. Cremer, "Theory of sound attenuation in a rectangular duct with an absorbing wall and the resultant maximum attenuation coefficient", Acustica 2, 249-263 (1953).

${ }^{28}$ T. Kato, Perturbation Theory for Linear Operators, 2nd edition, 623pp (Springer-Verlag, Berlin, Heidelberg) (1980).

${ }^{29}$ W. D. Heiss and A. L. Sannino, "Avoided level crossing and exceptional points", J. Phys. A 23, 1167 (1990).

${ }^{30} \mathrm{~W}$. D. Heiss, "Repulsion of resonance states and exceptional points", Phys. Rev. E 61, 929-932 (2000).

${ }^{31}$ M. Berry, "Physics of nonhermitian degeneracies", Czechoslovak Journal of Physics 54, 1039-1047 (2004).

${ }^{32}$ E. Hernández, A. Jáuregui, and A. Mondragón, "Energy eigenvalue surfaces close to a degeneracy of unbound states: Crossings and anticrossings of energies and widths", Phys.l Rev. E 72, 026221 (2005).

${ }^{33} \mathrm{~W}$. Bi and W. Pagneux, "New insights into mode behaviours in waveguides with impedance boundary conditions", arXiv:1511.05508 (2015)

${ }^{34} \mathrm{~W}$. Bi and V. Pagneux, "Resonance trapping" dans un guide traité par une impédance locale ("trapping resonance" in a locally reacting lined waveguide)", in CFA 2016, edited by S. F. d'Acoustique (Le Mans, France) (2016).

${ }^{35}$ L. Xiong, W. Bi, and Y. Aurégan, "Fano resonance scatterings in waveguides with impedance boundary conditions", J. Acoust. Soc. Am. 139, 764-772 (2016).

${ }^{36}$ B. Nennig, Y. Renou, and Y. Aurégan, "On the use of periodic inclusions embedded in porous lining to enhanced attenuation in waveguides", in Acoustics 2012 (Nantes, France) (2012).
${ }^{37}$ C. Engström, C. Hafner, and K. Schmidt, "Computations of lossy Bloch waves in two-dimensional photonic crystals", J. Comput. Theor. Nanosci. 6, 775-783 (2009).

${ }^{38}$ M. Collet, M. Ouisse, M. Ruzzene, and M. Ichchou, "Floquet-Bloch decomposition for the computation of dispersion of two-dimensional periodic, damped mechanical systems", Int. J. Solids Struct. 48, $2837-2848$ (2011).

${ }^{39} \mathrm{~F}$. Tisseur and K. Meerbergen, "The quadratic eigenvalue problem", SIAM Rev. 43, 235-286 (2001).

${ }^{40}$ E. Redon, A.-S. Bonnet-Ben Dhia, J.-F. Mercier, and S. Sari Poernomo, "Non-reflecting boundary conditions for acoustic propagation in ducts with acoustic treatment and mean flow", Int. J. Num. Meth. Eng. 86, 1360-1378 (2011).

${ }^{41}$ J. B. Lawrie and I. D. Abrahams, "An orthogonality relation for a class of problems with high-order boundary conditions; applications in sound-structure interaction", Q. J. Mech. Appl. Math. 52, 161-181 (1999).

${ }^{42}$ C. Geuzaine and J.-F. Remacle, "Gmsh: a three-dimensional finite element mesh generator with built-in pre- and postprocessing facilities", Int. J. Num. Meth. Eng. 79, 1309-1331 (2009).

${ }^{43} \mathrm{~F}$. Hecht, "New development in FreeFEM++", J. Numer. Math. 20, 251-265 (2012).

${ }^{44}$ V. Hernandez, J. E. Roman, and V. Vidal, "SLEPc: A scalable and flexible toolkit for the solution of eigenvalue problems", ACM Trans. Math. Software 31, 351-362 (2005).

${ }^{45}$ Y. Aurégan and M. Leroux, "Experimental evidence of an instability over an impedance wall in a duct with flow", J. Sound Vib. 317, 432-439 (2008).

${ }^{46}$ M. L. Munjal, Acoustics of ducts and Mufflers: with application to Exhaust and Ventilation System Design, 416, pp. 82 (John Wiley \& Sons) (1991). 